\title{
Noncontact surface tension measurement by drop rotation
}

\author{
Won-Kyu Rhim ${ }^{\text {a) }}$ and Takehiko Ishikawa ${ }^{\text {b) }}$ \\ Jet Propulsion Laboratory, California Institute of Technology, 4800 Oak Grove Drive, Pasadena, \\ California 91109
}

(Received 7 March 2001; accepted for publication 4 June 2001)

\begin{abstract}
Validity of the surface tension measurement technique that was proposed by Elleman et al. was experimentally verified. The technique was based on Brown and Scriven's work on the shape evolution of rotating drops. Molten tin and aluminum drops were levitated in high vacuum by the electrostatic levitator and rotated by applying a rotating magnetic field. This technique offers an alternative technique for those liquids where the drop oscillation technique cannot be used. As a demonstration, the technique was applied to a glass-forming alloy $\left(\mathrm{Zr}_{41.2} \mathrm{Ti}_{13.8} \mathrm{Cu}_{12.5} \mathrm{Ni}_{10.0} \mathrm{Be}_{22.5}\right)$ and its surface tension was measured down to the lower temperature where the drop oscillation technique could not be applied due to its high viscosity. (C) 2001 American Institute of Physics. [DOI: $10.1063 / 1.1388215]$
\end{abstract}

\section{INTRODUCTION}

The proposal of measuring surface tension based on drop rotation was first made by Elleman et al. ${ }^{1}$ Their proposal for surface tension measurement was based on the theoretical predictions on the drop shape evolution of rotating drops. The theoretical predictions of drop rotation processes have been brought to a rare degree of accuracy by Chandrasekhar, ${ }^{2}$ and by Brown and Scriven. ${ }^{3}$ These predictions have also been experimentally verified by a number of authors. Rhim et al. ${ }^{4}$ performed an experiment in a groundbased laboratory using a charged water drop suspended by an electrostatic levitator. They rotated the drop by exerting an acoustic torque, and observed the drop shape closely following the theoretical prediction. Wang et al. ${ }^{5}$ conducted their drop rotation experiment in the microgravity environment provided by the Space Shuttle (USML-1). Using drops of silicone oil and glycerin/water mixture, they obtained results that also closely agreed with the theoretical prediction when the drops rotated satisfying the solid-body-rotation condition. On the basis of these works, Elleman et al. proposed that the surface tension of a rotating liquid could be determined if the shape deformation at a known rotation frequency could be accurately measured. If their proposal can be verified, it will provide a way for surface tension measurement for those liquids where the drop oscillation technique cannot be used.

This method offers an alternate approach to the surface tension measurement in many liquids where the drop oscillation method can be used. ${ }^{6}$ However, for those liquids where the drop oscillation technique can no longer be used, such as in highly viscous liquids, this technique will be uniquely qualified to extract their surface tensions.

In this article validity of the proposed method will be demonstrated using molten metallic drops that were levitated

\footnotetext{
${ }^{a)}$ Present address: Materials Science Department, M/S 138-78, California Institute of Technology, Pasadena, CA 91125; electronic mail: wonkyu.rhim@caltech.edu

${ }^{b)}$ Present address: Space Utilization Research Center, NASDA, 2-1-1 Sengen Tsukuba, Ibaraki 305-8505, Japan.
}

in a high vacuum. In absence of gas friction, satisfying the solid-body-rotation condition can be warranted. After the basic principle is verified, it will be applied to a bulk glassforming alloy and measure the surface tension down to the temperatures where drop oscillation cannot be induced due to high viscosity.

\section{EXPERIMENTAL APPARATUS AND APPROACH}

The experiment was conducted using a high temperature electrostatic levitator (HTESL) that operated in high vacuum condition. The HTESL levitates a sample about $3 \mathrm{~mm}$ in diameter between a pair of parallel disk electrodes that are spaced about $12 \mathrm{~mm}$ (Fig. 1). The electric field between these two electrodes exerts electrostatic force on a charged sample and cancels the downward gravitational force. The four small side electrodes that surround the bottom electrode control the sample position along the horizontal directions. The vertical position control voltage is applied to the upper electrode, while the bottom electrode is electrically grounded through an alternating current (ac) voltage source. This ac voltage source generates oscillating electric field across the disk electrodes when it becomes necessary to induce resonant oscillations on the levitated drop. The methods of measuring surface tension and viscosity from oscillating drops that are levitated in the HTESL are described in detail in Ref. 6.

Two different methods can be used to induce a rotation on a levitated sample. The first method of generating a torque is to use photon pressure, ${ }^{7}$ and the second method is to apply a rotating magnetic field. ${ }^{8}$ While the first method could apply to a broad range of sample materials, the second method was used in the present experiment since it offered more precise rotation control for sample materials of high electric conductivity.

Mounted on the top electrode in Fig. 1 was a four-coil assembly that was designed to generate a horizontal magnetic field that rotated at an appropriate frequency $(400 \mathrm{~Hz}$ in the present experiment) around the vertical direction. The principle of the sample rotation mechanism is essentially the 


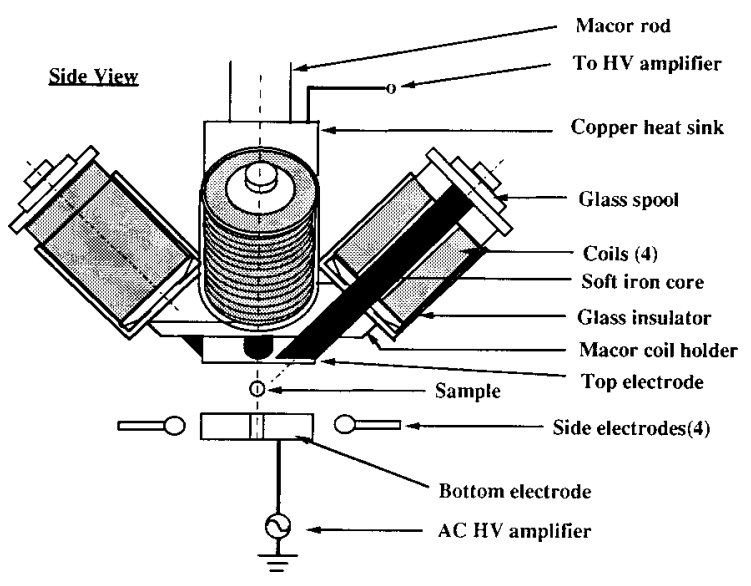

FIG. 1. Schematic side view of the electrode assembly. The four coil assembly designed to generate a rotating magnetic field is mounted on the top electrode, while the bottom electrode is connected to ground through a high voltage ac source to induce drop oscillations.

same as the asynchronous induction motor. The four-coil assembly plays the role of a stator while the levitated sample serves as a rotor. A more detailed description of the coil assembly and its performance can be found in Ref. 8. For this article it is sufficient to know that if an ac voltage $E_{1}$ at frequency $\omega_{s}$ is applied to a stator having a resistance $R_{1}$ and an inductance $L_{1}$, the torque $\tau$ experienced by the rotor having an instantaneous rotation frequency $\omega$ is given by

$$
\tau=\left(\frac{\omega_{s} E_{1}^{2}}{R_{1}^{2}+\omega_{s}^{2} L_{1}^{2}}\right) \frac{1}{R_{2}}\left(1-\frac{\omega}{\omega_{s}}\right)
$$

where $R_{2}$ and $L_{2}$ are, respectively, the resistance and the inductance of the rotor.

The electrode assembly was housed by a stainless steel chamber that was typically evacuated to $\sim 10^{-8}$ Torr. Samples were heated using a xenon arc lamp. A detailed description of the HTESL was given in an earlier publication. ${ }^{9}$

For sample rotation frequency measurement, a $\mathrm{He}-\mathrm{Ne}$ laser beam was directed to the sample, and the reflected beam was detected by a silicon photodetector. The output voltage of the detector was amplified and digitized to get a Fourier power spectrum. Such a power spectrum showed peaks at harmonics of sample rotation frequency. The power spectrum only served as a coarse indicator of sample rotation frequency. More precise detection of rotation frequency was achieved by exploiting the stroboscopic effects created by TV monitors. The frame rate of regular TV monitors is $30 \mathrm{~Hz}$ (or $60 \mathrm{~Hz}$ field rate). A charge coupled device camera operating at the shutter speed $0.001 \mathrm{~s}$ was mounted on a telemicroscope to produce a magnified sample image on a TV screen. Whenever the sample rotation rate approached one of the harmonics or subharmonics of the $60 \mathrm{~Hz}$ field rate, seemingly static images appeared on the screen. Such stroboscopic approach assisted by the power spectrum allowed unambiguous determination of sample rotation rate within 0.01 Hz.
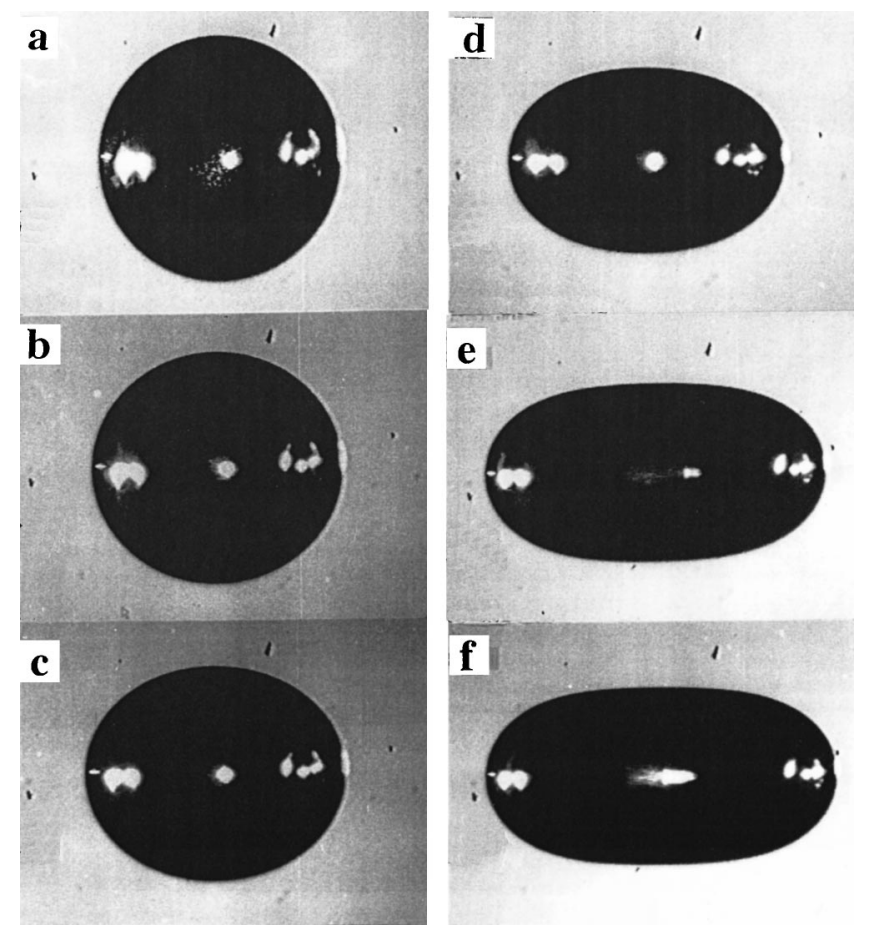

FIG. 2. Side views of a rotating aluminum drop as the angular momentum was progressively increased. The rotation frequencies of these figures are (a) 0, (b) 60, (c) 90, (d) 118.5, (e) 110.5, and (f) $108 \mathrm{~Hz}$, respectively. (d) Closely landed on the bifurcation point.

\section{DETERMINATION OF EFFECTIVE OSCILLATION FREQUENCY FROM THE SHAPES OF ROTATING DROPS}

The drop rotation experiment was conducted using tin and aluminum samples approximately $25 \mathrm{~K}$ above their melting points. When levitated drops were brought to stable nonrotating states, resonant oscillations $(n=2$ mode) were induced and the respective frequencies were recorded. Subsequently sample rotation was initiated by applying rotating magnetic field at a present amplitude and frequency.

As a drop started rotating around the vertical axis, the initial spherical shape progressively changed to oblate spherical shape, and this trend continued until the bifurcation point was reached at which the axisymmetric shape became unstable with respect to the triaxial ellipsoidal shape. After the transition to triaxial shape the drop rotation frequencies started to decrease due to rapidly increasing moment of inertia even though the drops gained angular momentum. Figure 2 shows the side views of a rotating aluminum drop. The view (a) shows the initial nonrotating drop, and the views (b), (c), and (d) depict progressively increasing oblateness along the axisymmetric branch. The view (e) shows the drop shape immediately following the bifurcation point, and the view (f) shows the drop shape with further elongated triaxial ellipsoidal shape corresponding to a further increased moment of inertia. Indeed, as shown in Fig. 3, this behavior was in accordance with the theoretical predictions.

In Fig. 3, the normalized drop dimension, $R_{\max } / R_{o}$, was plotted as a function of normalized rotation frequency, $\omega_{\text {rot }} / \omega_{\text {osc }}$, where $R_{\max }$ and $R_{o}$ were, respectively, the maximum radius of rotating drop and that of initial spherical drop. 


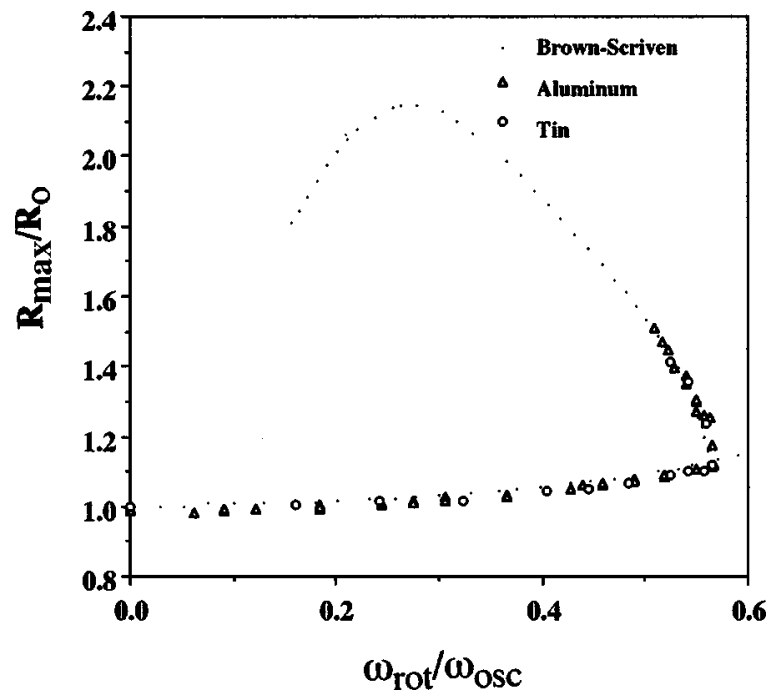

FIG. 3. Normalized drop dimension, $R_{\max } / R_{o}$, was plotted as a function of normalized rotation frequency, $\omega_{\text {rot }} / \omega_{\text {osc }}$, where $R_{\max }$ and $R_{o}$ were, respectively, the maximum radius of rotating drop and that of initial spherical drop. The drop rotation frequency $\omega_{\text {rot }}$ was normalized by its own oscillation frequency $\omega_{\text {osc }}(n=2$ mode $)$ at its nonrotating state. These experimental values are compared with the theoretical curves calculated by Brown and Scriven.

The drop rotation frequency $\omega_{\text {rot }}$ was normalized by its own oscillation frequency $\omega_{\text {osc }}(n=2$ mode $)$ at its nonrotating state. These experimental values are compared in the figure with the theoretical curves calculated by Brown and Scriven. ${ }^{3}$ Figure 3 shows that the experimental points of both the aluminum and tin drops follow the theoretical curve within $2 \%$. Also, the observed bifurcation points agrees with the predicted value, $\omega_{\text {rot }} / \omega_{\text {osc }}=0.559$, within $2 \%$. Transition between the axisymmetric and the triaxial branches was reversible as long as the applied torque level was kept sufficiently low to ensure the solid-body-rotation condition. We observe in Fig. 3 that the data points along the axisymmetric branch are slightly below the theoretical curve. This was caused due to slightly elongated spherical shape of the drops in their initial nonrotating states.

The fact that $\omega_{\text {rot }} / \omega_{\text {osc }}=0.559$ at the bifurcation point allows the indirect determination of the effective oscillation frequency $\omega_{\text {osc }}$ if the rotation frequency $\omega_{\text {rot }}$ is measured immediately past the bifurcation point. Then one can use $\omega_{\text {osc }}$ and determine the surface tensions of the sample following the procedure that was used in the familiar drop oscillation technique for surface tension measurement. ${ }^{6}$ In general, if $R_{\max } / R_{o}$ and $\omega_{\text {rot }}$ are measured, $\omega_{\text {osc }}$ can be determined from Fig. 3. Basically, this was the proposal made by Elleman et al. Indeed, in the present experiment, the rotation frequencies $\omega_{\text {rot }}$ of a molten tin drop could be measured along the axisymmetric branch using oxide patches on the drop surface as tracers.

The axisymmetric branch in Fig. 3 can be expressed by the following polynomial:

$$
\begin{aligned}
R_{\max } / R_{o}= & 1+1.476 \times 10^{-2} F+1.2532 F^{2}-1.7877 F^{3} \\
& +3.7385 F^{4},
\end{aligned}
$$

where $F=\omega_{\text {rot }} / \omega_{\text {osc }}$. Thus, this equation effectively deter-

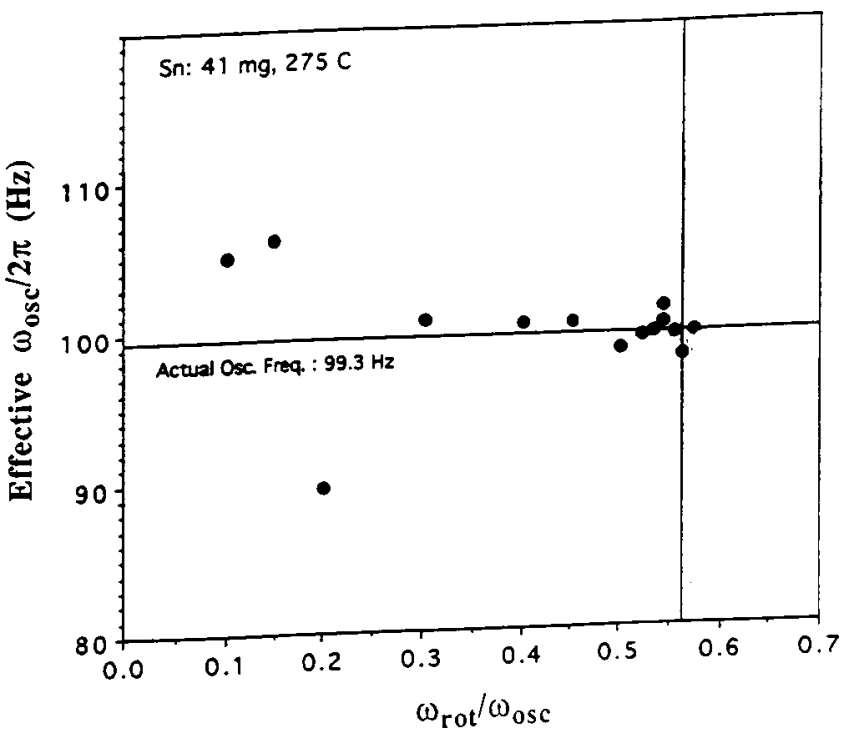

FIG. 4. The effective oscillation frequencies, $\omega_{\text {osc }} / 2 \pi$, of a molten tin drop are plotted as a function of rotation frequency. Also shown in the figure is the actual oscillation frequency of the sample that was actually measured at the same temperature.

mines what might be the oscillation frequency of the drop in its nonrotating state, $\omega_{\text {osc }}$, if drop dimension $R_{\max } / R_{o}$ is measured accurately at a given rotational frequency, $\omega_{\text {rot }}$. Figure 4 shows the effective oscillation frequency, $\omega_{\text {osc }} / 2 \pi$, of a molten tin drop so obtained as a function of rotation frequency. Also shown in the figure is the actual oscillation frequency of the sample that was measured at the same temperature. At the lower rotation frequency, the effective oscillation frequencies scattered widely, then, as the drop rotation frequency approached the bifurcation point, i.e., $\omega_{\text {rot }} / \omega_{\text {osc }}$ $=0.559$, the data points converged rapidly to the actual oscillation frequency. This can be understood since $R_{\max } / R_{o}$ increases with the rotation frequency, giving higher accuracy in $\omega_{\text {rot }} / \omega_{\text {osc }}$. From Fig. 4 one can see the effective oscillation frequency agreed with the actual frequency within $\pm 2 \%$ (which corresponded to $\pm 2 \mathrm{~Hz}$ ). This accuracy is lower than that of directly measured oscillation frequency that was reproducible within $0.5 \mathrm{~Hz}$. However, what makes this technique unique is in the fact that this noncontact technique can also measure the surface tension of highly viscous liquids for which the drop oscillation technique cannot be applied. We believe that the present accuracy of the effective frequency may be increased as the accuracy in drop sizing is improved. Finally, it is important to note that the present technique could measure the drop rotation frequency throughout the entire axisymmetric branch simply because the drop showed some surface structure. However, if sample surface does not offer any detectable structure, one should measure the drop rotation frequency immediately following the bifurcation point and use $\omega_{\text {rot }} / \omega_{\text {osc }}=0.559$ to determine the effective oscillation frequency $\omega_{\mathrm{osc}}$.

\section{APPLICATION TO A BULK GLASS-FORMING ALLOY}

For low viscosity liquids, the drop oscillation technique is an adequate noncontact technique for surface tension mea- 


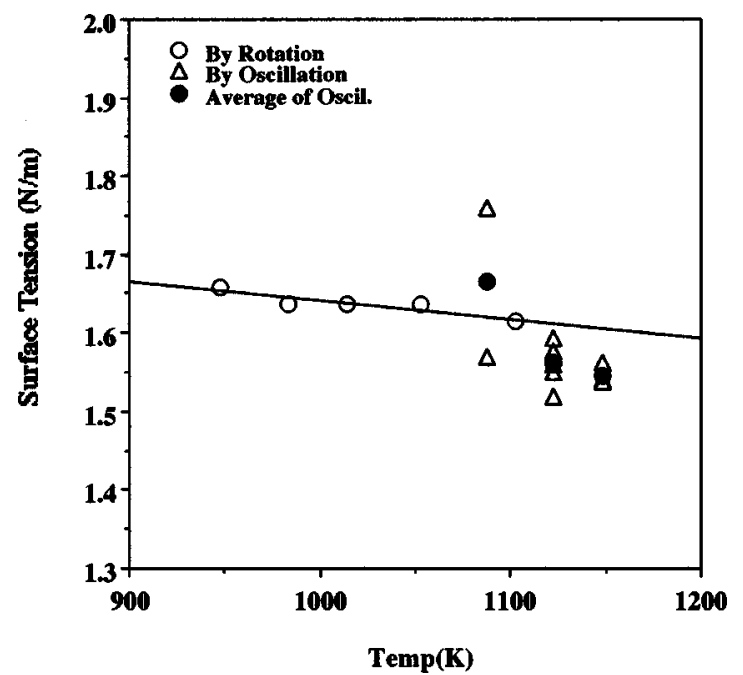

FIG. 5. Surface tensions of a bulk glass-forming alloy that were measured using the technique at several different temperatures. Also shown in the higher temperature range are the surface tension data obtained using the drop oscillation technique that was possible due to the lower liquid viscosity in this range.

surements. However, some liquids have such high viscosity that drop oscillations cannot be induced. For instance, viscosity of glass forming alloys increase nearly 14 orders magnitude as they approach their glass transition temperatures. In the present work a bulk glass-forming alloy $\left(\mathrm{Zr}_{41.2} \mathrm{Ti}_{13.8} \mathrm{Cu}_{12.5} \mathrm{Ni}_{10.0} \mathrm{Be}_{22.5}\right)^{10}$ was selected to demonstrate the capability of the new technique for surface tension measurement. The viscosity of this alloy increased so much below $1080 \mathrm{~K}\left(T_{m}=993 \mathrm{~K}\right)$ that it was not possible to induce oscillations on the levitated drop. Figure 5 shows the surface tensions of the glass-forming alloy that were measured with the technique. Also shown in the figure are the surface tension data that were obtained using the drop oscillation technique that was possible at the higher temperatures. The open circles are the surface tensions measured using the technique (the drop rotation method). The triangles are the data taken by the oscillation method and the solid circles are the averages of these data. Figure 5 shows that the drop oscillation method becomes increasingly less accurate as the sample temperature was lowered. However, the technique could take over and provided surface tension values at the lower temperatures that were impossible otherwise. The linear fit to the data can be expressed by

$$
S(T)=1.64-2.37\left(T-T_{m}\right) 10^{-4} \mathrm{~N} / \mathrm{m} \text {. }
$$

No data are available in the literature to compare with the present results.

\section{ACKNOWLEDGMENTS}

The authors would like to acknowledge Dr. PaulFrançois Paradis for his help in the data taking process and Daniel Barber for his technical support. One of the authors (T. I.) would like to thank NASDA of Japan for the support it provided during his one year stay at JPL. This work was carried out at the Jet Propulsion Laboratory, California Institute of Technology, under contract with the National Aeronautics and Space Administration.

${ }^{1}$ D. D. Elleman, T. G. Wang, and M. Barmatz, NASA Tech. Memo. 2, 557 (1988).

${ }^{2}$ S. Chandrasekhar, Proc. R. Soc. London, Ser. A 286, 1 (1965).

${ }^{3}$ R. A. Brown and L. E. Scriven, Proc. R. Soc. London, Ser. A 371, 331 (1980).

${ }^{4}$ W.-K. Rhim, S. K. Chung, and D. D. Elleman, AIP Conf. Proc. 197, 91 (1988).

${ }^{5}$ T. G. Wang, A. V. Anilkumar, C. P. Lee, and K. C. Lin, J. Fluid Mech. 276, 389 (1994).

${ }^{6}$ W. K. Rhim, K. Ohsaka, P.-F. Paradis, and R. E. Spjut, Rev. Sci. Instrum. 70, 2796 (1999).

${ }^{7}$ W. K. Rhim and P.-F. Paradis, Rev. Sci. Instrum. 70, 4652 (1999).

${ }^{8}$ W. K. Rhim and T. Ishikawa, Rev. Sci. Instrum. 69, 3628 (1998).

${ }^{9}$ W. K. Rhim, S. K. Chung, D. Barber, K. F. Man, G. Gutt, A. Rulison, and R. E. Spjut, Rev. Sci. Instrum. 64, 2961 (1993).

${ }^{10}$ A. Peker and W. L. Johnson, Appl. Phys. Lett. 63, 2342 (1990). 